\title{
A Paragenetic Perspective on Integration of RNA Silencing into the Epigenome and Its Role in the Biology of Higher Plants
}

\author{
R.A. Jorgensen, N. Doetsch, A. Müller, Q. Que, K. Gendler, And C.A. Napoli \\ Department of Plant Sciences, University of Arizona, Tucson, Arizona 85721-0036
}

\begin{abstract}
We describe features of RNA silencing and associated epigenetic imprints that illustrate potential roles for RNA interference (RNAi) in maintenance and transmission of epigenetic states between cells, throughout a plant, and perhaps even across sexual generations. Three types of transgenes can trigger RNAi of homologous endogenous plant genes: (1) "sense" transgenes that overexpress translatable transcripts, (2) inverted repeat (IR) transgenes that produce double-stranded RNA (dsRNA), and (3) antisense transgenes. Each mode of RNAi produces a different characteristic developmental silencing pattern. Single-copy transgenes are sufficient for sense-RNAi and antisense-RNAi, but not inverted repeat-RNAi. A single premature termination codon dramatically attenuates sense-RNAi, but it has no effect on antisense or inverted repeat-RNAi. We report here that antisense transgenes altered by removal of nonsense codons generate silencing patterns characteristic of sense-RNAi. Duplication of a sense overexpression transgene results in two types of epigenetic events: (1) complete loss of silencing and (2) altered developmental pattern of silencing. We also report that duplicating only the transgene promoter results in complete loss of silencing, whereas duplicating only transcribed sequences produces the second class, which are vein-based patterns. We infer that the latter class is due to systemic RNA silencing signals that interact with certain epigenetic states of the transgene to imprint it with information generated at a distance elsewhere in the plant.
\end{abstract}

R. Alexander Brink (1960) proposed that chromosomes possess two functions, one genetic and the other "paragenetic," the genetic components being stable and the paragenetic components being labile and programmable in ontogeny. Brink proposed calling the stable genetic components "orthochromatin" and the labile paragenetic components "parachromatin." Unlike euchromatin and heterochromatin, which are defined as alternative states and described as residing at distinct locations in the genome, orthochromatin (which Brink equated with DNA) and parachromatin (which Brink described as chromatin-protein complexes) are intimately associated with each other throughout the genome. The function of orthochromatin is to stably maintain and transmit genetic information, whereas the function of parachromatin is to mediate gene expression and to store programmable information regarding the history of a cell lineage at individual genetic loci. Thus, the terms paragenetic and parachromatin simply refer to epigenetic states that are chromosomally based. Brink consciously chose to propose the term paragenetic rather than use the term epigenetic because of the latter's broader and less specific definition in Waddington's (1942) original usage.

Today it is well established that the molecular basis for the lability of chromatin at individual loci is chromatin remodeling complexes that establish and maintain histone modifications, DNA modifications, nucleosome structure and positioning, and higher-order chromatin states (for review, see Mellor 2006). The recent recognition that components of the RNAi machinery participate in the establishment and maintenance of (at least some) chromatin states has expanded concepts of chromatin organization to include a broader role for noncoding RNA (ncRNA) molecules than was previously understood. ncRNA is an extremely abundant and diverse class of molecules, including not only microRNA (miRNA) and its precursors, but also antisense transcripts that often "readthrough" into annotated coding sequences, opposing "normal" sense transcripts. The resulting formation of dsRNA recruits the nuclear RNAi machinery to the locus, which then precipitates heterochromatin formation, involving histone and DNA modifications (Matzke and Birchler 2005).

Here, we describe features of RNA silencing and associated epigenetic imprints at chimeric Chalcone synthase $(C h s)$ transgene loci in petunia flowers that illustrate potential roles for RNAi in the maintenance and transmission of metastable states between cells, throughout a plant, and perhaps even across sexual generations.

\section{SENSE-RNAI AND THE ROLE OF MRNA TRANSLATION}

Three types of transgenes can trigger RNAi in plants: (1) "sense" transgenes that have been engineered to overproduce a translatable transcript, (2) inverted repeat (or "hairpin") transgenes that have been engineered to produce dsRNA transcripts, and (3) antisense transgenes, which are engineered to produce a transcript that is complementary to its target transcript (Jorgensen 2003). We refer to these three modes of transgene-induced RNAi as sense-RNAi, inverted repeat-RNAi, and antisense-RNAi, respectively. Sense-RNAi, originally known as sense cosuppression, results from attempted overexpression of a host protein (Napoli et al. 1990) and requires high levels of transcription of a translatable transcript (Que et al. 1997). A single premature termination codon (PTC) is sufficient to dramatically attenuate this mode of RNAi, indicating the importance of translation of the full-length coding region of the sense transcript. Sense-RNAi also 
requires a host RNA-dependent RNA polymerase (RdRP), which recognizes and copies overexpressed transcripts to produce the dsRNA molecules necessary to trigger RNAi (Beclin et al. 2002). Typical sense overexpression transgenes carry only the amino-acid-coding region of the target transcript and lack the target's $5^{\prime}$ - and 3'-untranslated regions (UTRs), as well as introns. However, none of these elements are necessary for silencing by single-copy transgenes (Q. Que et al., unpubl.).

Sense transgenes that have not been engineered for protein overproduction (e.g., see van der Krol et al. 1990) induce RNAi only when they are integrated in the genome as inverse repeats of the T-DNA element that carries the sense transgene and the selective marker from Agrobacterium into the plant genome (Que et al. 1997; Stam et al. 1997). Readthrough transcription of these long inverse repeats of T-DNA is thought to produce the double-stranded "foldback" RNA that triggers RNAi (Muskens et al. 2000). Because inverse repeat integrants may also produce dsRNA corresponding to the transgene promoter, stochastic epigenetic loss of silencing frequently occurs (Stam et al. 1998).

Single-copy antisense transgenes can trigger RNAi despite possessing multiple nonsense codons in all reading frames. Instead, they are thought to trigger RNAi by pairing with complementary target transcripts to form dsRNA. Silencing of anthocyanin pigment genes in petunia flowers by sense overexpression transgenes is visually distinct from antisense silencing. Sense-RNAi occurs as a developmental silencing pattern that is centered on the fusion zones between adjacent flower petals (Fig. 1a), whereas antisense transgenes never produce this pattern, instead producing palely pigmented flowers that may be blotchy and/or have white petal edges (Fig. 1b) (Napoli et al. 1990; Jorgensen et al. 1996; Que et al. 1998).

The inability of an antisense transgene to produce the developmental silencing pattern of sense overexpression transgenes could be due to the fact that the antisense transcript - even though it possesses a translation start codon in the same optimal translation context as the corresponding sense overexpression transcript-possesses several premature termination codons. To test this possibility, we engineered an antisense overexpression transgene by altering three nonsense codons to sense codons in the antisense strand of a 246-codon open reading frame (ORF) from a 740-bp segment of the petunia chalconesynthase-coding region; $20 \%$ of the transformants carrying this transgene exhibited the developmental silencing pattern characteristic sense-RNAi superimposed on the typical antisense phenotype (Fig. 1c,d). A control antisense construct retaining the three nonsense codons, and thus lacking a long ORF, did not produce any flowers with the sense-RNAi pattern.

An interesting technical aside is that the "nonsensecodons-removed" translatable antisense construct was more efficient than the control antisense plant at producing plants with completely white flowers. The nonsensecodon-removed antisense approach offers a potentially useful alternative to standard sense-RNAi and inverted repeat-RNAi. Silencing target genes by standard senseRNAi constructs has been avoided in crop genetic engineering because of the possibility that epigenetic loss of a
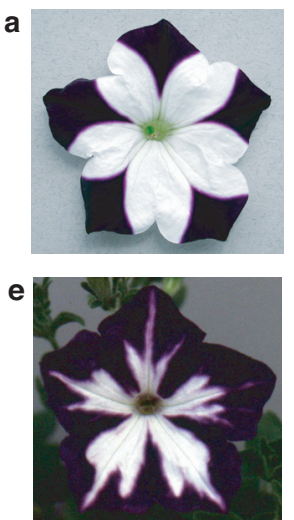

i. Primary transgene 35S Pro - ChsA - nos3'

Secondary transgene 35S Promoter duplication
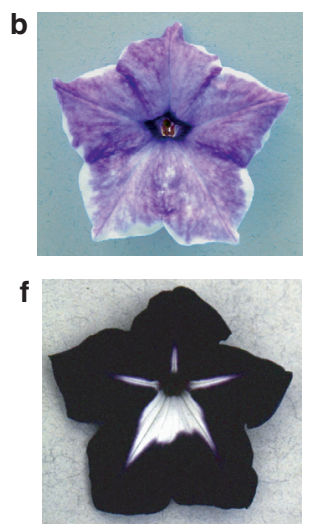

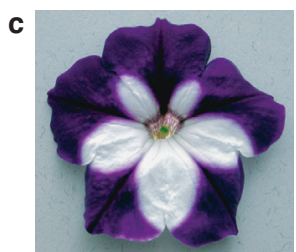

g

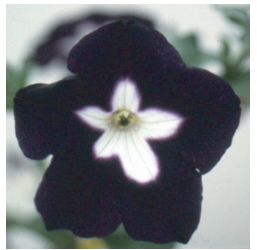

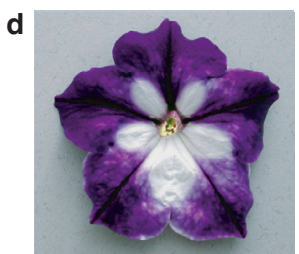

$\mathbf{h}$

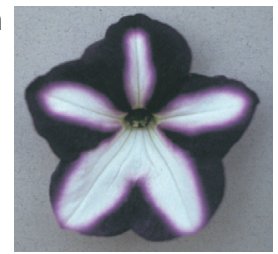

Flower Color Phenotype

Fusion zone pattern

Solid purple

Vein patterns

\section{ChsA - nos3' duplication}

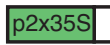

Figure 1. RNA silencing by Chs transgenes in petunia flowers. (a) Sense overexpression transgene (e.g., see "primary transgene" in $i$ ). (b) Antisense Chs transgene driven by $2 \times 35 \mathrm{~S}$. (c,d) Antisense Chs transgene with premature termination codons removed (see text). $(e, f, g)$ Three classes of vein-based patterns resulting from interaction between two transgene copies. $(h)$ Vein-based pattern induced by transcribed sequence duplication (see $i$, “ChsA-nos3' duplication"). (i) Chs transgene constructs. $2 \times 35 \mathrm{~S}$ is the cauliflower mosaic virus promoter with two copies of the upstream "enhancer" region. Chs $A$ refers to the coding sequence of the chalcone synthase gene. nos 3' refers to the 3'-untranslated region of the Agrobacterium tumefaciens nopaline synthase (nos) gene, which contains polyadenylation signals. (f,g, Reprinted, with permission, from Jorgensen 1995 [C AAAS].) 
the silent state would also result in overexpression of the target gene product by the sense transgene, as we have shown can occur with the petunia Hfl gene (Jorgensen et al. 2002). In contrast, inducing sense-RNAi with a translatable antisense transgene leaves no possibility of unintended overexpression of the target gene product.

\section{SENSE-TRANSGENE DUPLICATION INDUCES NOVEL EPIGENETIC STATES THAT INTERACT WITH SYSTEMIC SILENCING SIGNALS}

Duplication of a chalcone synthase transgene results in two classes of epigenetic change: (1) a complete loss of RNA silencing, resulting in uniformly purple flowers, and (2) a change in the morphological features that control the pattern of silencing, resulting in patterns of silencing based on the vasculature, i.e., vein-based patterns (Fig. 1e,f,g) (Jorgensen et al. 1996; Que and Jorgensen 1998). At least three heritably distinct types of vein-based patterns have been recognized (Jorgensen 1995; Jorgensen and Napoli 1996), indicating that multiple types of epigenetic events occur which are responsible for this class of silencing phenotypes.

By duplicating only the promoter of the overexpression transgene or by duplicating only its transcribed sequences (Fig. 1i), we found that the two classes of epigenetic changes are separable: (1) promoter duplication is responsible for the first class, loss of RNA silencing, and (2) duplication of the transcribed sequences is responsible for the second class, vein-based silencing patterns (example shown in Fig. 1h).

Interestingly, promoter duplication resulted in loss of silencing only with inverted repeat integrants of the secondary transgene (promoter-only construct). Presumably, this is because the loss of silencing is due to promoterderived dsRNA, and transgene inverted repeats are necessary for production of this dsRNA by transcriptional readthrough and transcript foldback, as discussed above. In contrast, single-copy integrants were sufficient to induce epigenetic changes when only transcribed sequences were duplicated (resulting in vein-based silencing patterns). Importantly, the vein-based silencing pattern state can also be generated by allelic interactions, demonstrating that ectopic location of the second transgene copy is not required. It is not clear how single-copy "promoterless" Chs transgenes induce epigenetic events; however, it is likely that such genes are transcribed from neighboring promoters, even if at low levels, and so it is conceivable that transcripts mediate epigenetic alteration of the target transgene, perhaps in RdRP-dependent fashion. In addition, the possibility remains that a direct DNA-DNA interaction between the two transgene copies is responsible.

\section{Paramutation}

Genetic segregation of the two transgene copies by outcrossing showed that the new vein-based silencing patterns could persist in the absence of the "promoterless" Chs transgene; thus, these epigenetic events are paramutations, according to Brink's broader, early definition. Interestingly, segregation of inverted repeat promoter transgenes away from the target sense overexpression Chs transgene also resulted in some plants with veinbased patterns. We would suggest that these vein-based patterns are due to "spreading" of the epigenetic state from the promoter of the sense transgene into the adjacent transcribed sequences, accompanied by loss or reduction in DNA methylation at the promoter in the absence of the inverted repeat promoter transgene.

Paramutation at the $b$ locus in maize is RNAi-mediated via an upstream array of tandem repeats (Alleman et al. 2006). Given that duplication of transcribed Chs sequences is sufficient to generate paramutations, as shown here, paramutation is probably not limited to transcriptional effects, nor to the presence of upstream repeats. Thus, an interesting possibility to consider is that naturally occurring alleles or paralogs of endogenous genes can interact via their transcribed regions to produce paramutation-like alterations in the chromatin of either or both gene copies.

\section{Systemic RNA Silencing Signals}

It is now well established that RNA and protein molecules can be trafficked through plasmodesmata in a regulated fashion and can even enter or leave the phloem stream via plasmodesmal connections to the phloem companion cells (Lucas and Lee 2004). This trafficking system has been referred to as the "RNA information superhighway" because it is potentially capable of longdistance transport of large numbers of informational macromolecules (Jorgensen et al. 1998). RNA silencing is also transmissible through plasmodesmata and the phloem and can spread through the tissues of an organ, as well as throughout the plant (Vaucheret 2006).

Our observation that duplication of transcribed sequences is responsible for patterns of silencing based on the vasculature suggests that these patterns are due to RNA silencing signals that emanate from the phloem of the major veins and move into the surrounding tissues via plasmodesmata. Two possibilities exist, either (1) the new epigenetic event (a paramutation) caused the transgene to create a phloem-transmissible RNA silencing signal that did not previously exist or (2) the new epigenetic state of the transgene is responsive to an RNA signal that was already present (or both). Because of the diversity of distinct, heritable epigenetic states that can arise from gene duplication (Fig. 1e,f,g), there could be (1) different types of RNA signals produced by the transgene depending on its epigenetic state, perhaps deriving from different or overlapping segments of the transcribed sequences, and/or (2) different responses, or efficiencies of response, of the transgene to the silencing signal that are determined by the epigenetic state of the transgene. Either way, it seems clear that epigenetic (paragenetic) states and systemic RNA silencing signals can interact.

\section{RNA-DIRECTED HETEROCHROMATINIZATION IN PLANTS}

Higher plants possess several novel classes of proteins that interact with the RNAi machinery in the formation of heterochromatin-like states. These include two novel 
types of DNA methyltransferases (chromomethyltransferase and domains-rearranged methyltransferase), a plant-specific clade of SNF2 ATPases, a large and diverse plant-specific clade of SET domain proteins related to Drosophila Su(var)3-9 and Schizosaccharomyces pombe clr4 and characterized by a novel plant-specific SRAYDG domain, plant-specific RNA polymerase subunits, which comprise a novel RNA polymerase IV, and plantspecific clades of Argonaute, Dicer-like, and RdRP proteins that participate in chromatin alterations and not miRNA silencing (Matzke and Birchler 2005).

Although plants, animals, and fungi share fundamental mechanisms of chromatin modification, such as histone methylation and acetylation, DNA methylation, ATPdependent nucleosome remodeling, and RNAi-mediated heterochromatin formation, higher plants appear to possess a more diversified complement of chromatin proteins involved in all these processes which may have been a basis for the evolution of novel plant-specific properties and functions of chromatin. Importantly, many of these novel forms of chromatin proteins are involved in RNAmediated alteration of chromatin, suggesting that higher plants could have a special capacity for chromatin remodeling involving RNA molecules (R.A. Jorgensen et al., in prep.).

\section{CONCLUSIONS}

R.A. Brink's (1960) suggestion that chromosomes possess a paragenetic function in addition to their genetic function and that the physical nature of this paragenetic function is a variety of forms or states of chromatin that can reside at any genetic locus is especially intriguing when considered in light of two important features of higher plants: (1) the evolutionary diversification of eukaryotic chromatin that appears to involve plantspecific mechanisms based on RNA and a diversified RNAi machinery, and (2) the capacity of plants to traffic informational macromolecules (RNA and protein) cell-tocell and systemically in a regulated manner (known as the "RNA information superhighway"). Given these capabilities, it is at least conceivable that higher plants may possess the capacity to store information at numerous genetic loci in the form of "paragenetic" chromatin states and that these states can be reprogrammed during ontogeny or in response to the environment.

As was shown here, paragenetic states appear to interact with systemic RNA silencing signals. Systemic trafficking of RNA (and protein) molecules could permit integration of paragenetic information over the whole of the organism, as well as differentiation of information states in different parts of the organism. Given the possibility of these events occurring at many thousands of genetic loci, such a system could operate as a high-capacity storage device that is reprogrammable during the life of the organism and reset each sexual generation.

If plants do possess such a system for information processing and storage that can integrate and assess information perhaps in large amounts and at both cellular and organismal levels, it would imply that plants might then be capable of making somewhat informed "decisions" to, for instance, fine-tune gene expression states during growth and development and in physiological responses to the environment. Such a means for information processing and decision making might be considered a form of "intelligence," but it would be one that is fundamentally different from the form of intelligence that evolved separately in animals for different purposes. For instance, timescales for the responsiveness of plant "intelligence" would be much slower than that of animal intelligence, given that plants generally have no possibility of relocating in response to the environment, whether in search of nutrients or to avoid predators. Instead, the "intelligence" of plants would be expected to address much longer timescales (e.g., diurnal) than are dictated by predator-prey interactions in animals. Furthermore, in long-lived perennials, plants' "memories" of past events could theoretically last for many years, and so could the implications of their "decisions." And finally, because plants do not sequester their germ lines, they also might conceivably pass on some of their paragenetically based memories to their offspring, possibly increasing their chances of survival and reproductive success.

\section{ACKNOWLEDGMENTS}

We thank Jin Wang and Jin-Xia Wu for their excellent technical assistance. This work was funded by grants to R.A.J. from the U.S. Department of Energy, Energy Biosciences Program (Award DE-FG03-98ER20308) and the National Science Foundation Plant Genome Research Program (Award 0421679), as well as a USDA postdoctoral fellowship to N.D.

\section{REFERENCES}

Alleman M., Sidorenko L., McGinnis K., Seshadri V., Dorweiler J.E., White J., Sikkink K., and Chandler V.L. 2006. An RNA-dependent RNA polymerase is required for paramutation in maize. Nature 442: 295.

Brink R.A. 1960. Paramutation and chromosome organization. Q. Rev. Biol. 35: 120.

Beclin C., Boutet S., Waterhouse P., and Vaucheret H. 2002. A branched pathway for transgene-induced RNA silencing in plants. Curr. Biol. 12: 684.

Jorgensen R.A. 1995. Cosuppression, flower color patterns, and metastable gene expression states. Science 268: 686.

. 2003. Sense cosuppression in plants: Past, present, and future. In RNAi: A guide to gene silencing (ed. G.J. Hannon), p. 5. Cold Spring Harbor Laboratory Press, Cold Spring Harbor, New York.

Jorgensen R.A. and Napoli C.A. 1996. A responsive regulatory system is revealed by sense suppression of pigment genes in Petunia flowers. In Genomes: Proceedings of the 22nd Stadler Genetics Symposium (ed. J.P. Gustafson and R.B. Flavell), p. 159. Plenum Press, New York.

Jorgensen R.A., Que Q., and Napoli C.A. 2002. Maternally controlled ovule abortion results from cosuppression of dihydroflavonol-4-reductase or flavonoid-3', 5'-hydroxylase genes in Petunia hybrida. Funct. Plant Biol. 29: 1501.

Jorgensen R.A., Atkinson R.G., Forster R.L., and Lucas W.J. 1998. An RNA-based information superhighway in plants. Science 279: 1486.

Jorgensen R.A., Cluster P.D., English J., Que Q., and Napoli C.A. 1996. Chalcone synthase cosuppression phenotypes in petunia flowers: Comparison of sense vs. antisense constructs and single-copy vs. complex T-DNA sequences. Plant Mol. Biol. 31: 957. 
Lucas W.J. and Lee J.Y. 2004. Plasmodesmata as a supracellular control network in plants. Nat. Rev. Mol. Cell Biol. 5: 712.

Matzke M.A. and Birchler J.A. 2005. RNAi-mediated pathways in the nucleus. Nat. Rev. Genet. 6: 24.

Mellor J. 2006. The dynamics of chromatin remodeling at promoters. Mol. Cell 19: 147.

Muskens M.W.M., Vissers A.P.A., Mol J.N.M., and Kooter J.M. 2000. Role of inverted DNA repeats in transcriptional and post-transcriptional gene silencing. Plant Mol. Biol. 43: 243.

Napoli C., Lemieux C., and Jorgensen R. 1990. Introduction of a chimeric chalcone synthase gene into petunia results in reversible co-suppression of homologous genes in trans. Plant Cell 2: 279.

Que Q. and Jorgensen R.A. 1998. Homology-based control of gene expression patterns in transgenic petunia flowers. Dev. Genet. 22: 100.

Que Q., Wang H.-Y., and Jorgensen R.A. 1998. Distinct patterns of pigment suppression are produced by allelic sense and antisense chalcone synthase transgenes in petunia flowers. Plant J. 13: 401.
Que Q., Wang H.-Y., English J., and Jorgensen R.A. 1997. The frequency and degree of cosuppression by sense chalcone synthase transgenes are dependent on transgene promoter strength and are reduced by premature nonsense codons in the transgene coding sequence. Plant Cell 9: 1357.

Stam M., Viterbo A., Mol J.N.M., and Kooter J.M. 1998. Position-dependent methylation and transcriptional silencing of transgenes in inverted T-DNA repeats: Implications for posttranscriptional silencing of homologous host genes in plants. Mol. Cell. Biol. 18: 6165.

Stam M., De Bruin R., Kenter S., Van der Hoorn R.A.L., Van Blokland R., Mol J.N.M., and Kooter J.M. 1997. Posttranscriptional silencing of chalcone synthase in Petunia by inverted transgene repeats. Plant J. 12: 63.

van der Krol A.R., Mur L.A., Beld M., Mol J.N.M., and Stuitje A.R. 1990. Flavonoid genes in petunia: Addition of a limited number of gene copies may lead to a suppression of gene expression. Plant Cell 2: 291.

Vaucheret H. 2006. Post-transcriptional small RNA pathways in plants: Mechanisms and regulations. Genes Dev. 20: 759

Waddington C.H. 1942. The epigenotype. Endeavour 1: 18. 


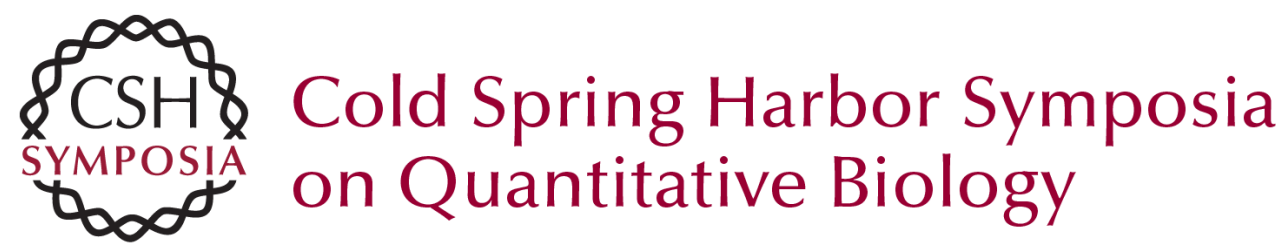

\title{
A Paragenetic Perspective on Integration of RNA Silencing into the Epigenome and Its Role in the Biology of Higher Plants
}

\author{
R.A. JORGENSEN, N. DOETSCH, A. MÜLLER, et al.
}

Cold Spring Harb Symp Quant Biol 2006 71: 481-485

Access the most recent version at doi:10.1101/sqb.2006.71.023

$\begin{array}{ll}\text { References } & \begin{array}{l}\text { This article cites } 20 \text { articles, } 7 \text { of which can be accessed free at: } \\ \text { http://symposium.cshlp.org/content/71/481.full.html\#ref-list-1 }\end{array}\end{array}$

\section{License}

Email Alerting Receive free email alerts when new articles cite this article - sign up in Service the box at the top right corner of the article or click here. 